\title{
A Universal Damping Function for Empirical Dispersion Correction on Density Functional Theory
}

\author{
Yi Liu* and William A. Goddard III* \\ Materials and Process Simulation Center (M/C 139-74), California Institute of Technology, \\ 1200 East California Blvd., Pasadena, California, USA 91125
}

\begin{abstract}
A damped London dispersion interaction is generally adopted in empirical dispersion corrections on density functional theory (DFT), where dispersion parameters are determined empirically to reproduce correct dispersive interactions after assuming a damping function. The key to a successful dispersion correction is choosing an appropriate damping function. In this work we propose a single universal damping function that can represent several damping functions used in literatures with a few adjustable parameters. This universal damping function provides a unified formula that allows convenient comparison and flexible optimization in dispersion corrected DFT methods. Using the optimized universal damping functions and dispersion parameters, we develop dispersion correction methods for HF, B3LYP and PBE theories. We calculate the dispersive energies accurately for rare gas diatomic molecules and benzene dimers with an averaged error $<4.1 \%$. [doi:10.2320/matertrans.MF200911]
\end{abstract}

(Received March 16, 2009; Accepted March 24, 2009; Published June 25, 2009)

Keywords: empirical dispersion correction, density functional theory, London dispersion, damping function

\section{Introduction}

Density functional theory (DFT) ${ }^{1,2)}$ becomes a popular quantum mechanics (QM) method in computational chemistry because of the reduced computational cost compared with $a b$ initio post Hartree-Fock (HF) quantum chemistry methods. Despite the great success of DFT in a wide range of applications, DFT failed to describe dispersive interactions in several cases as summarized below.

Pulay et $a l .{ }^{3)}$ employed GGA (BLYP) calculations on rare gas dimers $(\mathrm{He} 2, \mathrm{Ne} 2, \mathrm{Ar} 2)$, showing that BLYP yielded a purely repulsive interaction. Becke et al. ${ }^{4)}$ carried out LDA (PW), GGA (LDA+BP), GGA/exactX (B3LYP), and GGA (Bhalf-and-half) calculations on six rare gas dimers (He2, Ne2, Ar2, HeNe, HeAr, and NeAr). They showed that LDA severely overestimated dissociation energies, whereas GGA (LDA+BP) and GGA (B3LYP) yielded repulsive potential curves. Becke's half-and-half functional $(\mathrm{BH} \& \mathrm{H})$ accidentally led to weak bindings, but it still severely underestimated the dissociation energies. It was recently found ${ }^{5)}$ that $\mathrm{BH} \& \mathrm{H}$ qualitatively reproduced geometric and energetic details of parallel $\pi$-stacked aromatic complexes. However, this is presumably due to fortuitous error cancellation. Hobza et al. ${ }^{6)}$ applied BLYP, B3LYP, and B3P86 DFT methods to study H-bonded, ionic, electrostatic and London clusters. All these methods failed completely to describe London-type clusters, for which no minimum was found on the potential energy surfaces (PES). Jeong et al. ${ }^{7)}$ carried out SVWN, BLYP and B3LYP studies on X-Cl2 (van der Vaals complex) and X$\mathrm{HCl}$ (H-bonded complex) with large basis set $[6-311++\mathrm{G}$ (3df, 2pd)], and found that BLYP and B3LYP yielded much smaller binding energies than MP2 values. Sprik et al. ${ }^{8)}$ used LDA, LDA+B, LDA+B+LYP methods to study intermolecular interaction of benzene dimer. Consistent

*Corresponding authors, E-mails: yi@wag.caltech.edu, wag@wag.caltech. edu with previous studies of rare gas dimers, LDA exhibited a minimum on PES. However, this is fortuitous because the dispersive energy is not included in LDA. On the other hand, LDA $+\mathrm{B}$ and $\mathrm{LDA}+\mathrm{B}+\mathrm{LYP}$ yielded purely repulsive potentials.

The above brief review is not meant to be exhausted but should be convincing enough to prove the failure of DFT methods in those weakly interacting systems. The lack of dispersive interaction in DFT is a consequence of locality nature of the approximated exchange-correlation (xc) functionals adopted in DFT. It is inevitable to fix dispersion problems in DFT in order to study systems where the dispersive interaction plays an important role (e.g. biological molecules or molecular crystals). It has been shown that dispersive interactions can be incorporated into DFT quantum mechanically either through time-dependent DFT approaches $^{9,10)}$ or improved xc functionals. ${ }^{1-18)}$ These improved first principle approaches are often more expensive than the conventional DFT methods, which limit their applications in large scale simulations and dynamics studies. On the other hand, empirical dispersion corrections provide a pragmatic way to account for dispersive interactions in DFT without losing computational efficiency.

The empirical dispersion correction methods are main focus of this work. The idea of empirical dispersion correction is to compute damped dispersive interactions empirically and add them into conventional DFT energies as correction terms. Various correction methods have been proposed, differing mainly in the choice of damping functions. Detailed comparisons among different correction methods can be found in Sec. 2. In this work, we propose a new damping function that can represent various damping functions by using a single formula with different choice of parameters. This unified damping function allows convenient comparison among existing correction methods at an equal rooting. More importantly, the universal damping function can be utilized as a flexible formula for further improvements on dispersion corrected DFT methods. In Sec. 3, we 
demonstrate how the new universal damping function can be used to correct dispersions on DFT with examples of rare gas diatomic molecules and benzene dimers. Finally, we draw conclusions in Sec. 4.

\section{Methods of Dispersion Correction on DFT}

In the spirit of incorporating dispersive interaction into $\mathrm{HF},{ }^{19)}$ most current empirical dispersion corrected DFT (DFT-D) methods ${ }^{20-26)}$ augment a damped London dispersion $^{27)}$ energy into conventional DFT:

$$
E_{\mathrm{DFT}-\mathrm{D}}=E_{\mathrm{DFT}}+E_{\mathrm{disp}}
$$

where $E_{\mathrm{DFT}}$ is a normal DFT energy, and $E_{\mathrm{disp}}$ a dispersion correction term. The dispersion correction can be expressed in terms of a damped multipole expansion:

$$
\begin{aligned}
E_{\mathrm{disp}} & =-\sum_{i j, i<j}^{\mathrm{N}} \sum_{k=3}^{m} f_{\mathrm{damp}}\left(r_{i j}\right)\left(\frac{C_{2 k}}{r_{i j}^{2 k}}\right) \\
& =-\sum_{i j, i<j}^{\mathrm{N}} f_{\mathrm{damp}}\left(r_{i j}\right)\left(\frac{C_{6}}{r_{i j}^{6}}+\frac{C_{8}}{r_{i j}^{8}}+\frac{C_{10}}{r_{i j}^{10}}+\ldots\right) \\
& \approx-\sum_{i j, i<j}^{\mathrm{N}} f_{\mathrm{damp}}\left(r_{i j}\right) \frac{C_{6}}{r_{i j}^{6}}
\end{aligned}
$$

where $f_{\text {damp }}$ is a damping function. $C_{6}$ etc. are dispersion parameters for an atom type. The $C_{6}$ dispersion parameters between unlike atoms are calculated based on a geometrical mean $C_{\mathrm{ij}}=\left(C_{\mathrm{i}} C_{\mathrm{j}}\right)^{1 / 2} . \mathrm{N}$ is the number of subsystems (e.g. atom or molecule $i$ ). $r_{i j}$ is the distance between $i$ and $j$. The first sum runs over all pairs of subsystems, while the second sum represents a series of multipole expansion, describing the asymptotic behavior of dispersion. Higher order terms beyond $1 / r^{6}$ are neglected as an approximation in most current DFT-D methods.

Various DFT-D methods in literatures differ mainly in the choice of different damping functions. Wu and Yang ${ }^{20)}$ proposed two damping functions:

$$
f_{\text {damp }}\left(r_{i j}\right)=\left\{1-\exp \left[-c_{\text {damp }}\left(\frac{r_{i j}}{R_{i j}^{0}}\right)^{3}\right]\right\}^{2}
$$

where $c_{\text {damp }}=3.54$ was used. $R_{i j}^{0}$ is a sum of vdW radii of atom $i$ and $j$. Hereafter the damping function, eq. (3), is denoted as WY1 function. The other damping function is

$$
f_{\text {damp }}\left(r_{i j}\right)=\frac{1}{1+\exp \left[-\beta\left(\frac{r_{i j}}{R_{i j}^{0}}-1\right)\right]}
$$

where $\beta=23$.0. It was determined by requiring $f_{\text {damp }}=0.99$ at $r=1.2 \mathrm{R}_{0}$. Equation (4) is a Fermi function. Hereafter we denote this specific damping function as WY2 function. Wu and Yang concluded that WY1 was better than WY2 in their work because WY1 decays more slowly than WY2. However, Zimmerli et al. ${ }^{26)}$ obtained an opposite conclusion: WY2 was better for correcting HCTH, B3LYP, PW91 and PBE methods in the study of water benzene clusters.

WY2 damping function was further modified by Grimme et $a .^{21,22)}$ and Jurecka et al. $^{23)}$ by adding scaling factors.

$$
f_{\text {damp }}\left(r_{i j}\right)=\frac{s_{6}}{1+\exp \left[-d\left(\frac{r_{i j}}{s_{\mathrm{R}} R_{i j}^{0}}-1\right)\right]}
$$

Equation (5) has four parameters, namely, $s_{6}(\sim 1.0), d$ $(\sim 23), s_{\mathrm{R}}(\sim 1.0)$ and $R_{i j}^{0}$. $s_{6}$ is a global scaling parameter introduced by Grimme et al. $s_{\mathrm{R}}$ is a scaling parameter associated with xc functionals and the uncertainty of $\mathrm{vdW}$ radii. $d$ reflects the steepness of exponents. To correct some specific functionals, $s_{6}$ and $d$ need to be tuned. The damping function eq. (5) was used by Jurecka et al. ${ }^{23)}$ When $s_{\mathrm{R}}=1.0$, eq. (5) recovers Grimme's formula. ${ }^{21,22)}$ When $s_{6}=1.0$, $d=23, s_{\mathrm{R}}=1.0$, eq. (5) recovers WY2 function. Thus Grimme's and Jurecka's damping functions can be considered as variants of WY2 function.

Elstner et al. $^{24)}$ used the following damping function to correct an extended tight-binding method for dispersion:

$$
f_{\text {damp }}\left(r_{i j}\right)=\left\{1-\exp \left[-d\left(\frac{r_{i j}}{R_{i j}^{0}}\right)^{N}\right]\right\}^{M}
$$

where $d=3.0, N=7, M_{\circ}=4, R_{i j}^{0}=3.8 \AA$ for the first row elements, and $R_{i j}^{0}=4.8 \AA$ for the second row elements. Hereafter the damping function, eq. (6), is denoted as EHFSK function. Zimmerli et al. $^{26)}$ found EHFSK function was not as good as WY2 in their studies of water benzene clusters.

Ortmann et al. ${ }^{25)}$ used an exponential decay potential as follows:

$$
f_{\text {damp }}\left(r_{i j}\right)=1-\exp \left[-\lambda\left(\frac{r_{i j}}{R_{i j}^{0}}\right)^{n}\right]
$$

where $\lambda=7.5 \times 10^{-4}$ and $n=8$. These parameters were determined to reproduce the c-lattice constant of graphite. The damping function, eq. (7), is hereafter denoted as OBS function.

The literature review above shows that the damping functions currently used in the field vary substantially from function forms to parameters. The behaviors of chosen damping functions affect directly the quality of subsequent fitting of dispersion parameters. Thus it is critical to choose a good damping function in DFT-D methods. A damping function is normally designed with some constraints, e.g., it should approach to one at large distance but to zero at short distance. Despite these simple constraints, the choice of damping functions in literatures is somewhat arbitrary without rigorous justification and full optimization. Controversy exists in choosing good damping functions from different studies. ${ }^{20,26)}$

In this work we propose a new type of damping function as follows:

$$
f_{\text {damp }}\left(r_{i j}\right)=\left\{1+a \exp \left[-b\left(\frac{r_{i j}}{R_{i j}^{0}}\right)^{m}\right]\right\}^{n}
$$

where $a, b, m$ and $n$ are adjustable parameters. Hereafter we denote eq. (8) as a universal damping function. By choosing appropriate parameters in the universal damping function as shown in Table 1, we can reproduce the different damping functions that have been used in literatures. For example, when $a=\exp (23), b=23, m=1$, and $n=-1$, eq. (8) 
Table 1 Parameters used in the universal damping functions that represent various damping functions in literatures including WY $1,{ }^{20}$ WY $2,{ }^{20}$ ) $\mathrm{EHFSK}^{24)}$ and $\mathrm{OBS}^{25)}$ functions.

\begin{tabular}{llllr}
\hline & \multicolumn{1}{c}{$a$} & \multicolumn{1}{c}{$b$} & $m$ & $n$ \\
\hline WY1 & -1 & 3.54 & 3 & 2 \\
WY2 & $\exp (23)$ & 23 & 1 & -1 \\
EHFSK & -1 & 3 & 7 & 4 \\
OBS & -1 & 0.00075 & 8 & 1 \\
\hline
\end{tabular}
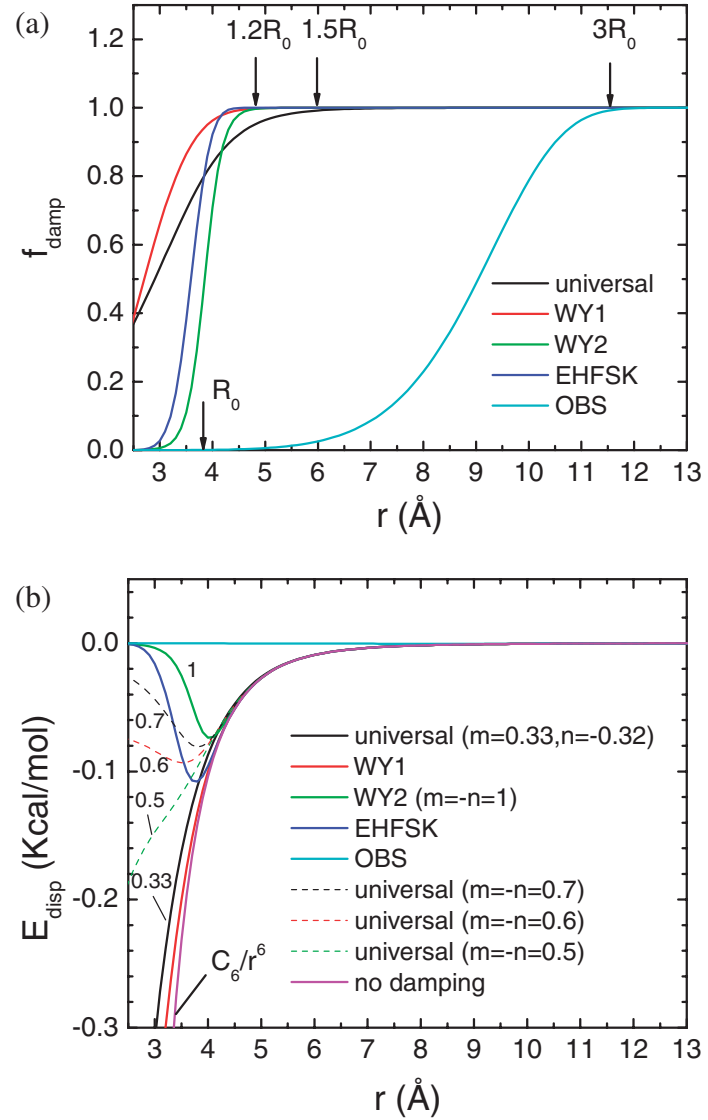

Fig. 1 (a) Optimized universal damping function [eq. (8)] and (b) dispersive correction developed in this work as well as those in literatures including WY $1^{20)}$ [eq. (3)], WY2 ${ }^{20)}$ [eq. (4)], $\mathrm{EHFSK}^{24)}$ [eq. (6)], and $\mathrm{OBS}^{25)}$ [eq. (7)]. The same C-C equilibrium bond distance $R_{0}=3.851 \AA$ and $C_{6}=425.765 \mathrm{kcal} / \mathrm{mol}^{6}$ are used in all dispersion corrections for the sake of comparison. The numbers labeled on the curves are the values of $\mathrm{m}$ used in the universal damping functions (see discussion in text).

recovers WY2 function. As an illustration, these damping functions and corresponding dispersion corrections are plotted as a function of distance between a pair of carbon atoms in Fig. 1(a) and 1(b), respectively.

To determine the parameters in the damping functions and dispersion parameters, we carried out the least square fitting to minimize the following error function:

$$
\begin{aligned}
\Delta E & =\sum_{i} w_{i}\left(E_{\text {exact }}-E_{\text {DFT-D }}\right)^{2} \\
w_{i} & =\exp \left[\left(E_{\min }-E_{i}\right) / k_{\mathrm{B}} T\right]
\end{aligned}
$$

where $w_{i}$ is a weighting factor related to the probability of finding a conformation in a canonical ensemble $(T=300 \mathrm{~K})$. $E_{\text {min }}$ is an energy minimum and $k_{\mathrm{B}}$ is a Boltzmann constant. $E_{\text {exact }}$ can be either an experimental value or a high level $a b$ initio quantum chemistry result. The parameter optimization was done by a conjugate gradient algorithm with full analytical derivatives, implemented in Computational Materials Design Facility $(\mathrm{CMDF})^{28)}$ developed in Caltech. $\mathrm{CMDF}$ is a flexible and extendable framework that integrates various multi-paradigm multi-scale methods using Python scripting language. The dispersion module and QM module in CMDF were used to compute dispersion and QM energies in this work.

\section{Results and Discussions}

\subsection{Rare gas diatomic molecules}

Rare gas diatomic molecules are bound through pure dispersive interactions, serving ideal benchmark systems for studying dispersive interactions with least ambiguity. We first calculated the binding energies of rare gas diatomic molecules including $\mathrm{He}, \mathrm{Ne}, \mathrm{Ar}, \mathrm{Kr}$, and $\mathrm{Xe}$ using $\mathrm{HF}$, B3LYP, ${ }^{29)}$ and $\mathrm{PBE}^{30}$ methods implemented in Jaguar ${ }^{31)}$ package. Unrestricted HF and DFT methods were used with $6-311 \mathrm{G}^{* *}++$ basis set and fine grid for numerical integrations. The basis set superposition error (BSSE) was corrected with Boys and Bernardi's counterpoise (CP) method. ${ }^{32)}$ The large basis set and BSSE correction are necessary to minimize the numerical uncertainty in the study of these weakly bound systems. The pseudospectrum method in Jaguar was not used in order to make our parameters transferable to work with other QM packages. The calculated QM binding energies (BE) as a function of atom separation are plotted in Fig. 2 in comparison with experimental values. ${ }^{33,34)}$ The calculated equilibrium distances and binding energy minima are tabulated in A1 of Appendix. We found that both HF and B3LYP led to positive energy curves, indicating that there are no energy minima on the PES. PBE yielded weak bindings, but still severely underestimated the experimental BE except for $\mathrm{Ne}-\mathrm{Ne}$. On the other hand, PBE predicted too large equilibrium distance.

We optimized both the universal damping function and dispersion parameters in order to reproduce the experimental $\mathrm{BE}$ curves. The resulting dispersion energies $\left(E_{\mathrm{disp}}\right)$ and DFT-D energies $\left(E_{\text {DFT-D }}\right)$ are plotted in Fig. 2. By adding dispersion corrections, DFT-D binding energies are in very good agreement with experimental values at a wide range of separations for various rare gas dimers. The optimized parameters used in these dispersion corrections are tabulated in $\mathrm{A} 2$ of Appendix.

\subsection{Benzene dimer}

Benzene dimer is the simplest prototype system for studying aromatic $\pi-\pi$ interactions that play an important role in biological systems (e.g. DNA base pairs). Benzene dimers have several typical configurations, namely, $\mathrm{T}$ shaped, sandwich, and parallel-displaced configurations. The schematic illustrations of these configurations are shown in the inserts of Fig. 3. The high-level $a b$ initio $\operatorname{CCSD}(\mathrm{T})^{35)}$ calculations were carried out for these benzene dimers with aug-cc-pVQZ* basis set and counterpoise correction. Coupled-cluster theory takes into account electron correlation, thus it is capable of describing dispersive interaction accurately. These $\operatorname{CCSD}(\mathrm{T})$ results serve as references for 

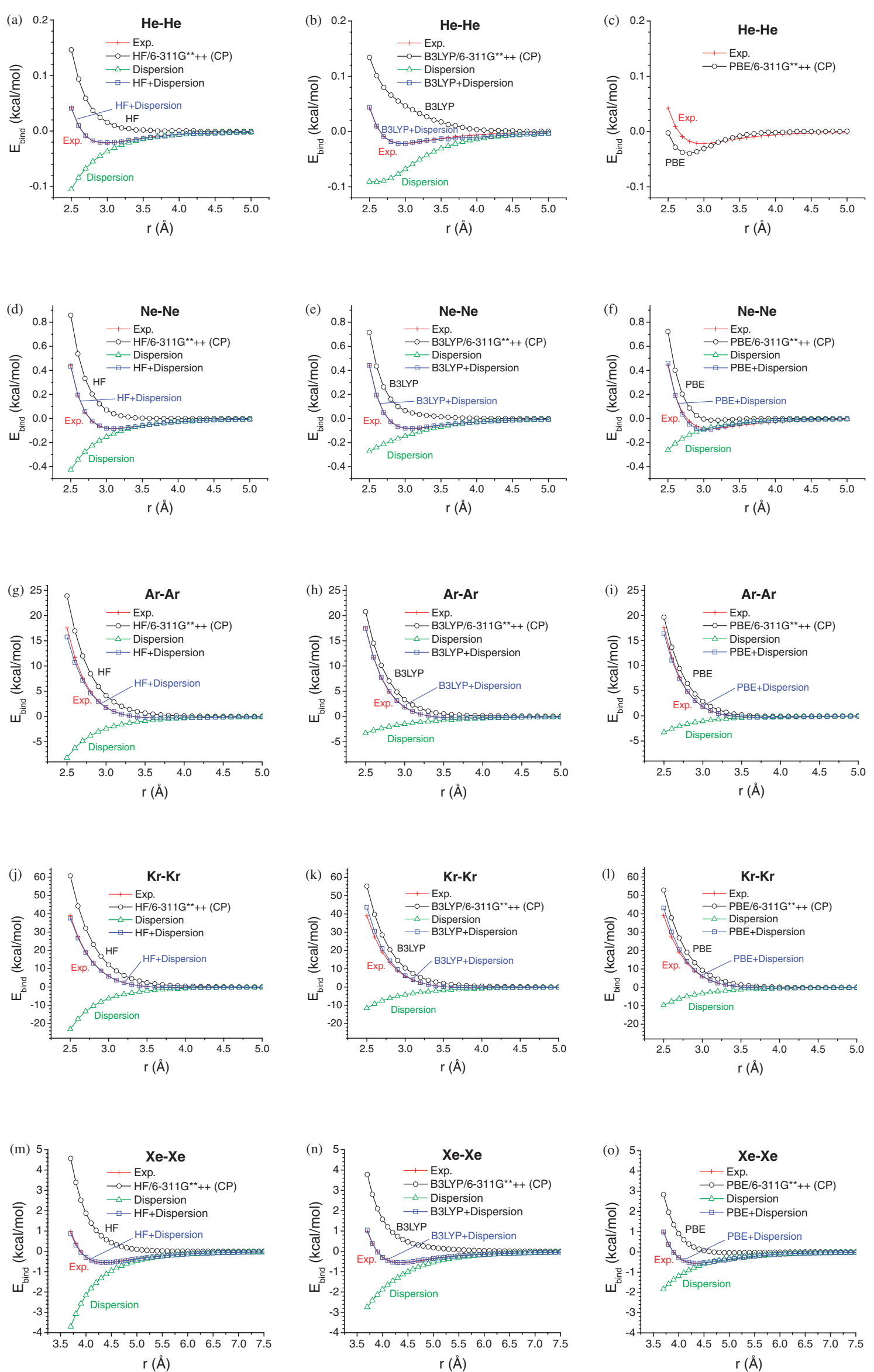

Fig. 2 Binding energies of rare gas diatomic molecules as a function of distance calculated using pure HF, B3LYP, and PBE as well as their dispersion corrections: (a)-(c) He-He, (d)-(f) Ne-Ne, (g)-(i) Ar-Ar, (j)-(l) Kr-Kr, (m)-(o) Xe-Xe. 

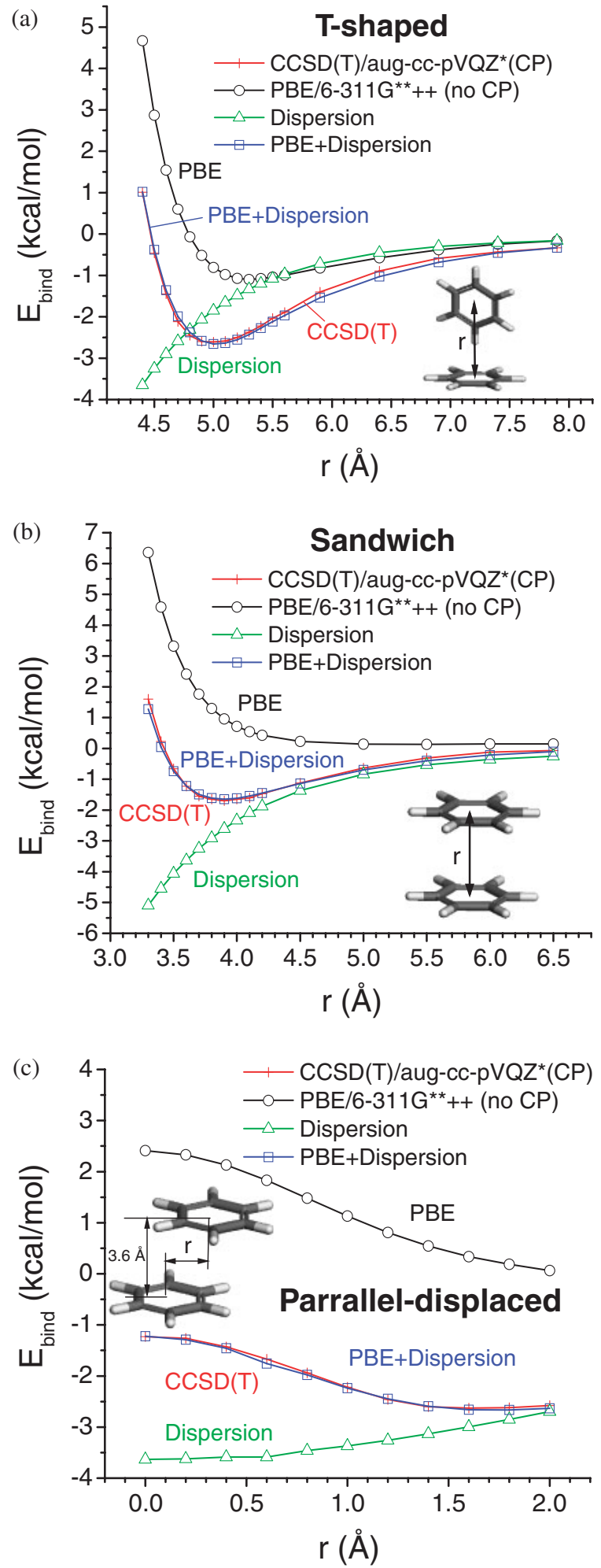

Fig. 3 Binding energies of benzene dimers as a function of distance calculated using pure PBE and its dispersion correction (PBE-D) for (a) Tshaped, (b) sandwich, (c) parallel-displaced configurations as shown in the inserts.

our dispersion corrections. The $\operatorname{CCSD}(\mathrm{T})$ calculations found that the parallel-displaced and the T-shaped configurations were almost energetically degenerate, and more stable than the sandwich configuration by 0.93 and $0.91 \mathrm{kcal} / \mathrm{mol}$, respectively (see Table 2). This can be understood since the $\mathrm{C}-\mathrm{H}$ electrostatic interactions dominate in the T-shaped
Table 2 Equilibrium distances $\left(R_{\min }\right)$ and binding energy minima $\left(E_{\min }\right)$ of benzene dimers calculated using $\mathrm{PBE}$ methods and its dispersion correction (PBE-D). $R_{\text {min }}$ has a unit of $\AA$, $E_{\text {min }} \mathrm{kcal} / \mathrm{mol}$.

\begin{tabular}{|c|c|c|c|c|c|c|}
\hline & \multicolumn{2}{|c|}{ T-shaped } & \multicolumn{2}{|c|}{ sandwich } & \multicolumn{2}{|c|}{ parallel-displaced } \\
\hline & $R_{\text {min }}$ & $E_{\min }$ & $R_{\min }$ & $E_{\min }$ & $R_{\min }$ & $E_{\min }$ \\
\hline $\operatorname{CCSD}(\mathrm{T})^{*}$ & 5.0 & -2.610 & 3.9 & -1.700 & 1.6 & -2.630 \\
\hline PBE & 5.3 & -1.100 & 5.5 & 0.129 & - & - \\
\hline PBE-D & 5.0 & -2.656 & 3.9 & -1.644 & 1.8 & -2.667 \\
\hline
\end{tabular}

*ab initio QM data are taken from Ref. 35).

configuration, whereas the weaker dispersive interactions dominate in the sandwich configuration. The electrostatic and the dispersive interactions coexist in the parallel-displaced configuration.

We calculated the binding energies of benzene dimers for all three configurations using DFT-PBE method implemented in Jaguar since our calculations on rare gas diatomic molecules show that PBE performances the best in describing dispersion among the studied methods. The $6-311 \mathrm{G}^{* *}++$ basis set and fine grid were used in the PBE calculations. Counterpoise correction was not included in this case because the CP correction is not practical for large systems, and we want our dispersion parameters to be applicable to most routine DFT calculations. The pseudospectrum method in Jaguar was not used for parameter transferability. Figure 3 shows the PBE binding energies together with the CCSD(T) results for comparison. As seen from Table 2, PBE severely underestimated the binding energies for the T-shaped and sandwich configurations compared to the $\operatorname{CCSD}(\mathrm{T})$ results. The equilibrium distances calculated by PBE were too long. No energy minimum was found on the PES of the paralleldisplaced configuration.

With a single set of parameters in the dispersion corrections, our PBE-D reproduced the $\operatorname{CCSD}(\mathrm{T})$ results well for three different configurations at a wide range of separations as shown in Fig. 3 and Table 2. These configurations of benzene dimers exhibit distinct binding characteristics changing from dispersive to electrostatic interactions, thus they serve as good test cases to examine the transferability of the dispersion parameters. Table 3 lists the optimized parameters used in the dispersion corrections with the universal damping functions.

During the least square fitting process we started from the WY2 damping function $[a=\exp (23), b=23, m=1$ and $n=1$ ], and optimized six parameters simultaneously, namely, $a, b, m, n$ and $C_{6}$ for $C$ and $H$. The resulting optimized damping parameters are $a=\exp (23), b=23.01434, m=$ 0.33360 and $n=-0.31952$. The $m$ and $n$ are about $1 / 3$ of the initial values, whereas $a$ and $b$ change little. This optimized universal damping function and corresponding dispersion corrections are plotted in Fig. 1(a) and 1(b) in comparison with those used in literatures. As shown in Fig. 1(a), the optimized universal damping function starts to decay $\left(f_{\text {damp }}=0.99\right)$ at $r=1.5 R_{0}$. For comparisons, WY1, WY2 and EHFSK functions were designed to decay at $r=1.2 R_{0}$. OBS function starts to decay at $r=3 R_{0}$. The slope of the universal damping function is similar to that of OBS function, but smaller than those of WY1, WY2 and EHFSK functions. From Fig. 1(b), we found that the dispersion 
Table 3 Parameters used in the dispersion corrections for benzene dimers in this work. PBE-D represents dispersion corrections on a PBE method. $R_{0}$ has a unit of $\AA, C_{6} \mathrm{kcal} / \mathrm{mol} \AA^{6} . a, b, m$ and $n$ are dimensionless.

\begin{tabular}{|c|c|c|c|c|c|c|c|c|}
\hline & \multirow[b]{2}{*}{$a$} & \multirow[b]{2}{*}{$b$} & \multirow[b]{2}{*}{$m$} & \multirow[b]{2}{*}{$n$} & \multicolumn{2}{|l|}{$\mathrm{C}$} & \multicolumn{2}{|l|}{$\mathrm{H}$} \\
\hline & & & & & $R_{0}^{*}$ & $C_{6}$ & $R_{0}^{*}$ & $C_{6}$ \\
\hline PBE-D & $\exp (23)$ & 23.01434 & 0.33360 & -0.31952 & 1.9255 & 425.747 & 1.4400 & 5.487 \\
\hline
\end{tabular}

${ }^{*}$ vdW radii are taken from Ref. 36 ).

correction using the universal damping function behaves similarly to that using WY1 function, even though the fitting started initially from the very different WY2 function. To conform the possibility of this transition, we examined the behaviors of universal damping functions by varying $\mathrm{m}$ $(=-n)$ from $0.7,0.6$, to 0.5 while fixing $a=\exp (23)$ and $b=23$. These universal damping functions are plotted as dashed lines in Fig. 1(b). We indeed observed the gradual changes from WY2 to the universal function when $\mathrm{m}$ decreases. Our optimization results are consistent with the conclusion of $\mathrm{Wu}$ and $\mathrm{Yang}^{20)}$ that the soft damping WY1 is better than the hard damping WY2. Our optimized damping function has a different function form but a similar effect as WY1 on dispersion corrections at the studied range. $\mathrm{Wu}$ and Yang found the better damping function by comparing two fixed functions. In this work, we obtained the similarly behaved damping function naturally through optimization.

\section{Conclusions}

Designing a good damping function is critical in developing a successful empirical dispersion corrected DFT method. We here propose a universal damping function that unifies different damping functions that have been used in literatures. By implementing the single universal function, one can examine and compare the performances of various existing DFT-D methods at an equal footing. Moreover, the flexibility of the universal function allows further optimization of damping functions, e.g., a smooth change of the softness of damping. The optimization of damping function is not only a mathematical obligation but also an estimation of missing dispersive interactions in DFT methods. It is possible to design a variant of the universal damping function by imposing additional physical constraints. Applying dispersion corrections on DFT using the optimized universal damping functions, we can describe dispersive interactions accurately for rare gas diatomic molecules and benzene dimers. It is straightforward to develop parameters for other atom types in different chemical environments.

\section{Acknowledgments}

We acknowledge the supports from ARO (W911NF-05-10345) and ONR (N00014-05-1-0778).

\section{REFERENCES}

1) P. Hohenberg and W. Kohn: Phys. Rev. B 136 (1964) B864.

2) W. Kohn and L. J. Sham: Phys. Rev. 140 (1965) 1133.

3) S. Kristyan and P. Pulay: Chem. Phys. Lett. 229 (1994) 175.

4) J. M. Perezjorda and A. D. Becke: Chem. Phys. Lett. 233 (1995) 134.

5) M. P. Waller, A. Robertazzi, J. A. Platts, D. E. Hibbs and P. A. Williams: J. Comput. Chem. 27 (2006) 491.

6) P. Hobza, J. Sponer and T. Reschel: J. Comput. Chem. 16 (1995) 1315.

7) H. Y. Jeong and Y. K. Han: Chem. Phys. Lett. 263 (1996) 345.

8) E. J. Meijer and M. Sprik: J. Chem. Phys. 105 (1996) 8684.

9) W. Kohn, Y. Meir and D. E. Makarov: Phys. Rev. Lett. 80 (1998) 4153 .

10) A. Hesselmann and G. Jansen: Chem. Phys. Lett. 367 (2003) 778.

11) Y. Zhao and D. G. Truhlar: Phys. Chem. Chem. Phys. 7 (2005) 2701.

12) X. Xu and W. A. Goddard: Proc. National Academy Sci. U.S. 101 (2004) 2673.

13) A. D. Becke and E. R. Johnson: J. Chem. Phys. 127 (2007) 154108.

14) Y. Zhang, X. Xu and W. A. Goddard: Proc. National Academy Sci. U.S. 106 (2009) 4963.

15) M. Dion, H. Rydberg, E. Schroder, D. C. Langreth and B. I. Lundqvist: Phys. Rev. Lett. 92 (2004) 246401.

16) A. Puzder, M. Dion and D. C. Langreth: J. Chem. Phys. 124 (2006) 164105.

17) O. A. von Lilienfeld, I. Tavernelli, U. Rothlisberger and D. Sebastiani: Phys. Rev. Lett. 93 (2004) 153004.

18) A. Tkatchenko and O. A. von Lilienfeld: Phys. Rev. B 73 (2006) 153406.

19) R. Ahlrichs, R. Penco and G. Scoles: Chem. Phys. 19 (1977) 119.

20) Q. Wu and W. T. Yang: J. Chem. Phys. 116 (2002) 515.

21) J. Antony and S. Grimme: Phys. Chem. Chem. Phys. 8 (2006) 5287.

22) S. Grimme, J. Antony, T. Schwabe and C. Muck-Lichtenfeld: Org. Biomol. Chem. 5 (2007) 741.

23) P. Jurecka, J. Cerny, P. Hobza and D. R. Salahub: J. Comput. Chem. 28 (2007) 555.

24) M. Elstner, P. Hobza, T. Frauenheim, S. Suhai and E. Kaxiras: J. Chem. Phys. 114 (2001) 5149.

25) F. Ortmann, F. Bechstedt and W. G. Schmidt: Phys. Rev. B 73 (2006) 205101.

26) U. Zimmerli, M. Parrinello and P. Koumoutsakos: J. Chem. Phys. 120 (2004) 2693.

27) F. London: Z. Phys. Chem. Abt. B11 (1930) 22.

28) M. J. Buehler, A. C. T. van Duin and W. A. Goddard: Phys. Rev. Lett. 96 (2006) 95505.

29) A. D. Becke: J. Chem. Phys. 98 (1993) 5648.

30) J. P. Perdew, K. Burke and M. Ernzerhof: Phys. Rev. Lett. 77 (1996) 3865.

31) Jaguar, version 7.0, Schrodinger, LLC, New York, NY, (2007).

32) S. F. Boys and F. Bernardi: Mol. Phys. 19 (1970) 553.

33) J. F. Ogilvie and F. Y. H. Wang: J. Mol. Stru. 273 (1992) 277.

34) J. F. Ogilvie and F. Y. H. Wang: J. Mol. Stru. 291 (1993) 313.

35) M. O. Sinnokrot and C. D. Sherrill: J. Phys. Chem. A 108 (2004) 10200.

36) A. K. Rappe, C. J. Casewit, K. S. Colwell, W. A. Goddard and W. M. Skiff: J. Am. Chem. Soc. 114 (1992) 10024.

37) A. Bondi: J. Chem. Phys. 68 (1964) 441. 


\section{Appendix}

Table A.1 Equilibrium distances $\left(R_{\min }\right)$ and binding energy minima $\left(E_{\min }\right)$ of rare gas diatomic molecules ( $\mathrm{He}, \mathrm{Ne}, \mathrm{Ar}, \mathrm{Kr}$ and $\mathrm{Xe}$ ) calculated using $\mathrm{HF}$, B3LYP and PBE methods as well as their corresponding dispersion corrections (HF-D, B3LYP-D, and PBE-D). $R_{\min }$ has a unit of $\AA, E_{\min }$ $\mathrm{kcal} / \mathrm{mol}$.

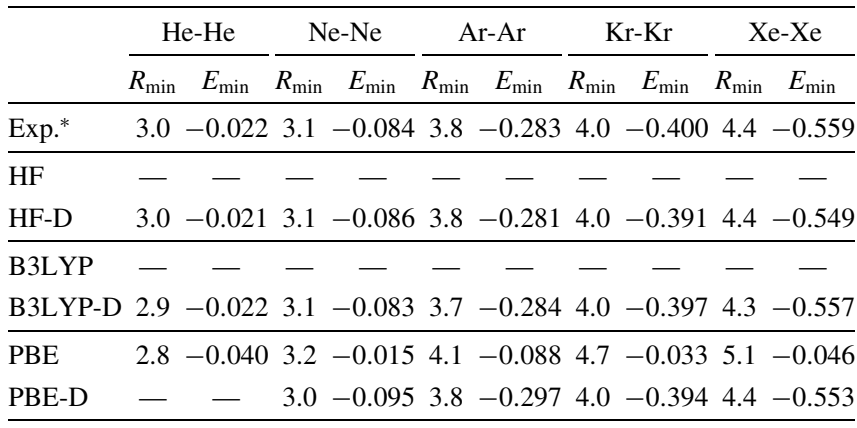

*Experimental data are taken from Ref. 33) and 34).
Table A.2 Parameters used in the dispersion corrections for rare gas diatomic molecules ( $\mathrm{He}, \mathrm{Ne}, \mathrm{Ar}, \mathrm{Kr}$ and $\mathrm{Xe}$ ) in this work. HF-D, B3LYP$\mathrm{D}$ and PBE-D represent dispersion corrections on HF, B3LYP and PBE methods. $R_{0}$ has a unit of $\AA$ A, $C_{6} \mathrm{kcal} / \mathrm{mol} \AA^{6}$. $a, b, m$ and $n$ are dimensionless.

\begin{tabular}{lcccccc}
\hline \multicolumn{1}{c}{$a$} & $b$ & $m$ & $n$ & $R_{0}{ }^{*}$ & $C_{6}$ \\
\hline He-He & & & & & & \\
HF-D & $\exp (23)$ & 23.36785 & 0.33650 & -0.06361 & 1.40 & 27.262 \\
B3LYP-D & $\exp (23)$ & 23.15017 & 0.66830 & -0.54746 & 1.40 & 57.050 \\
PBE-D & - & - & - & - & - & - \\
\hline Ne-Ne & & & & & & \\
HF-D & $\exp (23)$ & 23.32193 & 0.31384 & -0.07403 & 1.54 & 115.762 \\
B3LYP-D & $\exp (23)$ & 22.92656 & 0.73908 & -0.19545 & 1.54 & 129.379 \\
PBE-D & $\exp (23)$ & 23.17713 & 0.04913 & -0.27044 & 1.54 & 77.750 \\
\hline Ar-Ar & & & & & & \\
HF-D & $\exp (23)$ & 23.11613 & 0.07113 & 0.71417 & 1.88 & 973.286 \\
B3LYP-D & $\exp (23)$ & 22.30994 & 0.39826 & -0.21014 & 1.88 & 1890.334 \\
PBE-D & $\exp (23)$ & 23.26067 & -0.05601 & -0.48332 & 1.88 & 956.537 \\
\hline Kr-Kr & & & & & & \\
HF-D & $\exp (23)$ & 23.06095 & 0.06604 & 1.17749 & 2.02 & 1587.617 \\
B3LYP-D & $\operatorname{exp(23)~}$ & 23.57374 & 2.57724 & -0.01571 & 2.02 & 3646.323 \\
PBE-D & $\operatorname{exp(23)~}$ & 23.50139 & -0.16150 & -0.10775 & 2.02 & 2392.454 \\
\hline Xe-Xe & & & & & & \\
HF-D & $\operatorname{exp(23)~}$ & 23.01312 & 0.06445 & 1.15343 & 2.16 & 3742.731 \\
B3LYP-D & $\exp (23)$ & 23.85045 & 0.90806 & -0.07369 & 2.16 & 8366.453 \\
PBE-D & $\operatorname{exp(23)~}$ & 23.52811 & 1.58400 & -0.01384 & 2.16 & 5035.130 \\
\hline
\end{tabular}

${ }^{*}$ vdW radii are taken from Ref. 37 ). 